CARDIOVASCULAR MEDICINE

\title{
Myocardial late gadolinium enhancement cardiovascular magnetic resonance in hypertrophic cardiomyopathy caused by mutations in troponin I
}

\author{
J C Moon, J Mogensen, P M Elliott, G C Smith, A G Elkington, S K Prasad, D J Pennell, W J McKenna
}

Heart 2005;91:1036-1040. doi: 10.1136/hrt.2004.041384

See end of article for authors' affiliations

\section{Correspondence to:}

Professor William j

McKenna, Cardiology in

the Young, The Heart

Hospital, University

College London Hospitals

Trust, 16-18

Westmoreland Street

London WIG 8PH, UK;

william.mckenna@uclh.org

Accepted

13 September 2004
Objective: To examine the influence of genotype on late gadolinium enhancement (LGE) and the potential of cardiovascular magnetic resonance (CMR) to detect preclinical hypertrophic cardiomyopathy.

Design: Prospective, blinded cohort study of myocardial LGE in a genetically homogeneous population.

Patients: 30 patients with disease causing mutations in the recognised hypertrophic cardiomyopathy gene for cardiac troponin I (TNNI3): 15 with echocardiographically determined left ventricular hypertrophy $(\mathrm{LVH}+)$ and 15 without (LVH-).

Main outcome measures: CMR measures of regional left ventricular function, wall thickness, and mass, and the extent and distribution of LGE.

Results: LGE was found in 12 (80\%) LVH+ patients but with variable extent (mean 15\%, range 3-48\%). LGE was also found in two (13\%) LVH- patients but the extent was limited (3.6\%) and both patients were found to have an abnormal ECG and regional hypertrophy by cine CMR. The extent of LGE was positively associated with clinical markers of sudden death risk $(21 \%$ with $\geqslant 2$ risk factors $v 7 \%$ with $\leqslant 1$ risk factor, $\mathrm{p}=0.02)$ and left ventricular mass $(r=0.56, \mathrm{p}<0.001)$ and was inversely associated with ejection fraction $(r=-0.58, p<0.001)$. Segmental analysis showed that as regional wall thickness increased, LGE was more prevalent ( $p<0.0001)$ and more extensive $(r=0.98, p=0.001)$.

Conclusion: In patients with disease causing mutations in TNNI3, focal fibrosis was not detected by LGE CMR before LVH and ECG abnormalities were present. Once LVH is present, LGE is common and the extent correlates with adverse clinical parameters. This suggests that focal fibrosis is closely linked to disease development.
$\mathrm{H}$ ypertrophic cardiomyopathy (HCM) is a clinically and genetically heterogeneous disease. HCM can be defined in a variety of ways: genetically by the presence of disease causing mutations in any of 10 genes encoding sarcomeric proteins ${ }^{1}$; and clinically by the presence of unexplained cardiac hypertrophy. ${ }^{2}$ Histologically, HCM is characterised by the presence of extensive myocardial disarray and various patterns of myocardial fibrosis. ${ }^{3}$ The link between genetic mutation and phenotype development including risk of sudden death and the development of heart failure is poorly understood but the environment, sex, and modifier genes ${ }^{56}$ are important in causing progressive myocardial abnormality characterised by hypertrophy, myocardial fibrosis, and disarray. ${ }^{78}$

It has not been possible to comprehensively quantify myocardial fibrosis and disarray in vivo' but a new technique, late gadolinium enhancement (LGE) cardiovascular magnetic resonance $(\mathrm{CMR})$, shows promise in this regard. ${ }^{10}{ }^{11}$ Regions of myocardial LGE, found after a gadolinium contrast agent injection, represent regions of increased myocardial fibrosis, and allow a direct assessment of the underlying myocardial abnormality. ${ }^{12}$ Previous studies in patients with ungenotyped HCM have shown correlations of LGE extent with clinical risk of sudden death and presence of heart failure. ${ }^{10}$ However, it is not known whether in these studies the LGE found reflects primarily genetic or non-genetic contributions or whether LGE would be useful for the detection of early, preclinical disease.

In this study, we examined a genotyped cohort, all of whom carried mutations in the cardiac troponin I gene (TNNI3)..$^{13}$ We hypothesised that the presence of LGE would be useful to detect early disease and would remain linked to clinical risk of sudden death despite the presence of mutations in the same disease causing gene.

\section{METHODS}

\section{Genotyped patients}

As part of an ongoing gene identification programme in an established HCM clinic, 748 unrelated patients were screened for mutations in the cardiac troponin I gene, as previously reported. ${ }^{13}$ A total of 23 families were identified with 100 mutation carriers, 48 of whom fulfilled established diagnostic criteria for HCM. ${ }^{2}$ The evidence that the mutations are disease causing was previously published and is essentially as follows: firstly, the amino acid substitution identified in probands was present in all relatives with the disease; secondly, no sequence variations led to amino acid substitutions in 150 ethnically matched control chromosomes; thirdly, identical mutations appeared in several unrelated families; and lastly, all mutations were located in functionally important and conserved regions of the gene. ${ }^{12-15}$ All living patients without contraindications to CMR in the UK from this cohort were invited to participate. Patients with all 13 mutations within the main study participated. Thirty patients were recruited: 15 with unexplained left ventricular hypertrophy $(\mathrm{LVH})$ on echocardiography $(\mathrm{LVH}+)$ and 15 gene positive relatives without $(\mathrm{LVH}-)$.

Abbreviations: CMR, cardiovascular magnetic resonance; DTPA, diethylenetriaminepentaacetic acid; $\mathrm{HCM}$, hypertrophic cardiomyopathy; LGE, late gadolinium enhancement; LVH, left ventricular hypertrophy 
Table 1 Distribution of risk factors for sudden death and clinical characteristics of patients with $(\mathrm{LVH}+)$ and without $(\mathrm{LVH}-)$ left ventricular hypertrophy

\begin{tabular}{lll}
\hline Characteristic & LVH+ (n= 15) & LVH- (n=15) \\
\hline Age (years) & $38(17)$ & $29(17)$ \\
$\quad$ Range & $8-67$ & $11-53$ \\
Male sex & $10(67 \%)$ & $8(53 \%)$ \\
Number with $\geqslant 2$ risk factors for & & \\
sudden death & 8 & 0 \\
Abnormal ECG & $15(100 \%)$ & $3(20 \%)$ \\
NYHA class & & \\
I & $8(53 \%)$ & $15(100 \%)$ \\
II & $6(40 \%)$ & 0 \\
$\quad 1$ III & $1(7 \%)$ & 0 \\
Family history of HCM & $4(27 \%)$ & $9(60 \%)$ \\
+ sudden death & $2(13 \%)$ & $1(7 \%)$ \\
Unexplained syncope & $2(13 \%)$ & $1(7 \%)$ \\
Non-sustained VT on Holter & $1(7 \%)$ & 0 \\
Sustained VT/VF & $6(40 \%)$ & $2(13 \%)$ \\
Abnormal exercise BP & $4(27 \%)$ & 0 \\
Maximum LVWT $\geqslant 30$ mm &
\end{tabular}

Values are shown as mean (SD) or number (\%).

BP, blood pressure; HCM, hypertrophic cardiomyopathy; LVWT, left ventricular wall thickness; NYHA, New York Heart Association heart failure class; VF, ventricular fibrillation; VT, ventricular tachycardia.

\section{Patient phenotype characterisation}

All patients at entry into the HCM clinic underwent 12 lead ECG, two dimensional echocardiography, and stratification for risk factors for sudden death according to five standard criteria. Five clinical risk factors for sudden death were used to stratify patients: a family history of HCM and two or more family members with sudden premature ( $\leqslant 40$ years) cardiac death; unexplained syncope; non-sustained ventricular tachycardia defined as three or more ventricular extrasystoles at a rate of 120 beats/min on ambulatory ECG; an abnormal blood pressure response (defined as a failure of systolic blood pressure to rise by more than $25 \mathrm{~mm} \mathrm{Hg}$ from baseline values or a fall of more than $15 \mathrm{~mm} \mathrm{Hg}$ from the maximum blood pressure) during upright exercise testing in $\leqslant 40$ year olds; and the presence on echocardiography of severe $\mathrm{LVH}(\geqslant 30 \mathrm{~mm}) .{ }^{16}$ This assessment was repeated at entry into this study to assess disease progression and to ensure correct stratification into LVH+ or LVH - categories. All echocardiography was performed by experienced operators in the dedicated HCM clinic and reviewed at entry into the study.

\section{CMR}

CMR was performed as previously described. ${ }^{10}$ Briefly, a short axis stack of cine images was acquired to assess global and regional function. A bolus of $0.1 \mathrm{mmol} / \mathrm{kg}$ gadoliniumdiethylenetriaminepentaacetic acid (DTPA) was administered intravenously and LGE imaging was started at five minutes with an inversion recovery technique, meticulous adjustment of the inversion time, a $90^{\circ}$ pre-saturation pulse over the cerebrospinal fluid to eliminate ghosting, and imaging parameters optimised according to the patient's breath hold ability and heart rate. Images with potential LGE were repeated with a phase swap for confirmation.

\section{Image analysis}

All data were analysed blinded to the clinical details. The cine images were quantified by planimetry to determine myocardial mass. The maximum diastolic segmental wall thickness was measured. When present, LGE was quantified for extent and this was expressed as a percentage of the total myocardial mass. Segments were analysed in a 16 segment ( six base, six mid, and four apex) model, ${ }^{17}$ with both diastolic wall thickness and LGE expressed as quintiles: $\leqslant 9 \mathrm{~mm} ; 10$ $14 \mathrm{~mm}, 15-19 \mathrm{~mm} ; 20-24 \mathrm{~mm} ; \geqslant 25 \mathrm{~mm}$; no LGE, $\leqslant 24 \%$; $25-49 \% ; 50-74 \%$; $\geqslant 75 \%$.

\section{Statistical analysis}

Non-parametric Wilcoxon rank comparisons were used to compare LGE extent in patients with fewer $(\leqslant 1)$ versus more $(\geqslant 2)$ risk factors for sudden death. Regression was used to compare total extent of LGE with continuous variables (age, left ventricular mass, ejection fraction, and

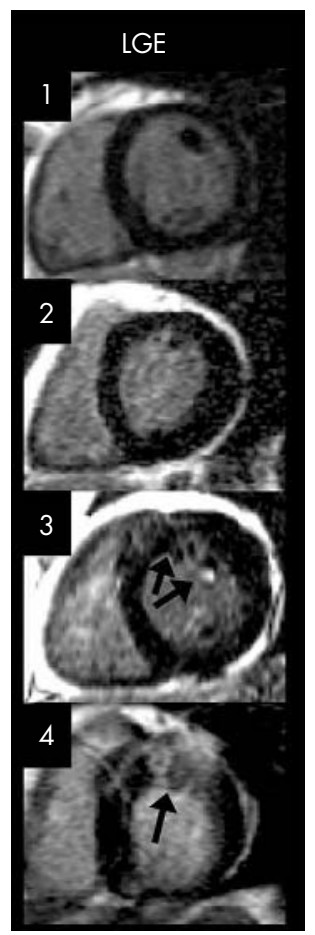

\begin{tabular}{|c|c|}
\hline $400^{\top}$ & \\
\hline EF & $74 \%$ \\
\hline Mass & 57 \\
\hline Cine/LGE & $\mathrm{n} / 0 \%$ \\
\hline Echo & $\mathrm{n}$ \\
\hline ECG & $\mathrm{n}$ \\
\hline $490^{\pi}$ & \\
\hline $\mathrm{EF}$ & $68 \%$ \\
\hline Mass & 71 \\
\hline Cine/LGE & $a b n / 0 \%$ \\
\hline Echo & $\mathrm{n}$ \\
\hline ECG & $a b n$ \\
\hline $530^{\pi}$ & \\
\hline EF & $71 \%$ \\
\hline Mass & 72 \\
\hline Cine/LGE & $a b n / 3 \%$ \\
\hline Echo & $\mathrm{n}$ \\
\hline ECG & $a b n$ \\
\hline $51 \delta^{\pi}$ & \\
\hline EF & $72 \%$ \\
\hline Mass & 141 \\
\hline Cine/LGE & $a b n / 6 \%$ \\
\hline Echo & $a b n$ \\
\hline SG & $a b n$ \\
\hline
\end{tabular}

Figure 1 Progression of late gadolinium enhancement (LGE) in a family with more than four gene positive members: H805 (Arg 145Trp), a family with late onset, low risk disease without progression during follow up and little LGE. abn, abnormal; CMR, cardiovascular magnetic resonance; $\mathrm{EF}$, ejection fraction; $n$, normal.

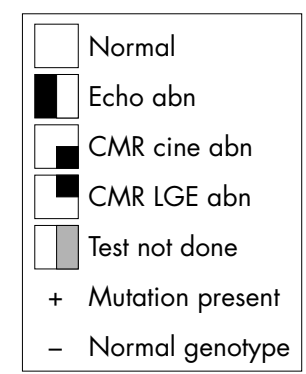



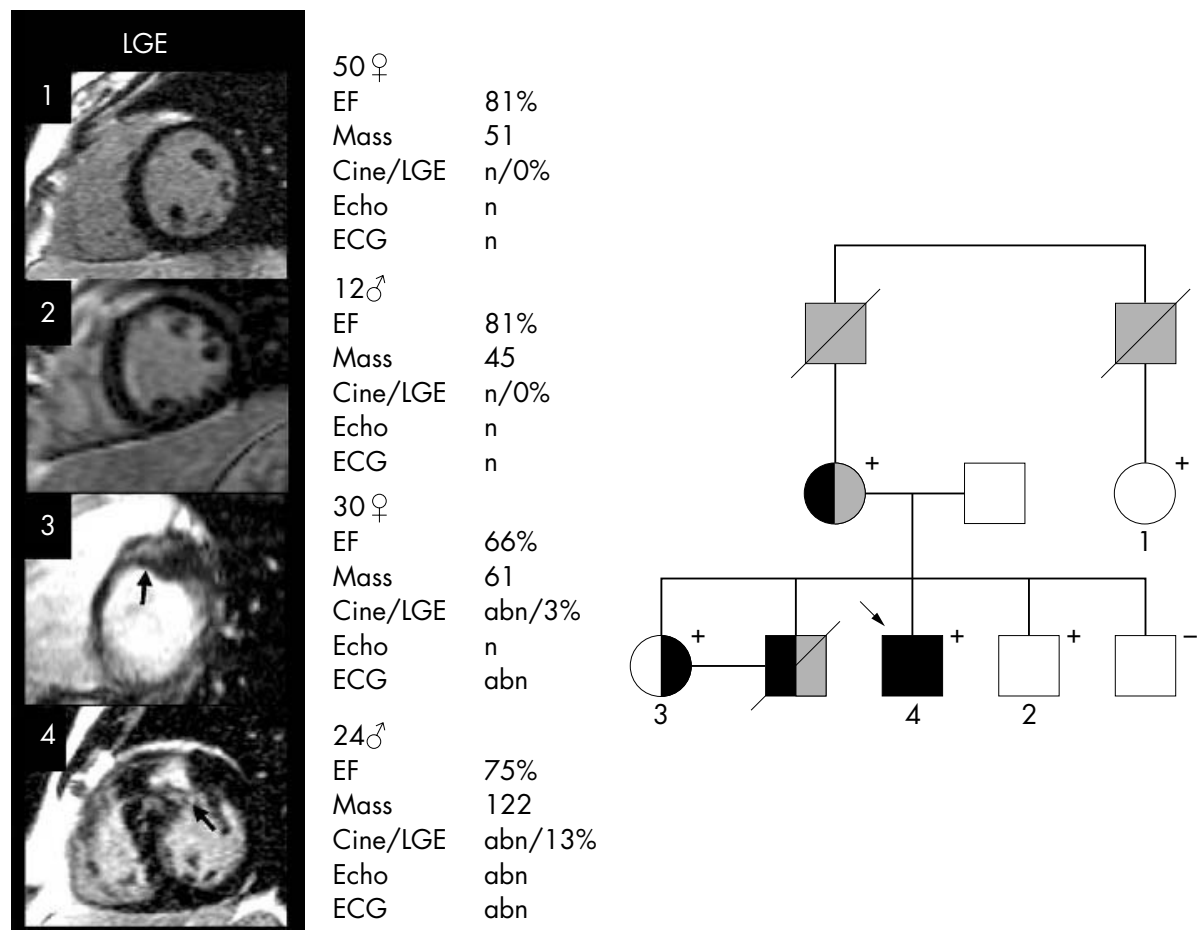

Figure 2 Progression of LGE in a family with more than four gene positive members. $\mathrm{H} 15$ (Arg162Gln), a family with early onset disease (in males) with rapid phenotype development, significant enhancement in the hypertrophied regions, and significant risk of sudden death.

wall thickness). The segmental presence of LGE and wall thickness were compared by $2 \times \mathrm{n}$ contingency table analysis.

\section{RESULTS}

\section{Patient baseline characteristics}

Table 1 gives patient baseline characteristics (at time of scanning). Eleven patients had asymmetrical hypertrophy (three with outflow obstruction), one in a dilated phase, two with apical HCM, and one with severe restrictive physiology.

\section{Phenotype development before CMR}

During clinic follow up before CMR (mean 49 months), phenotype had changed in five patients. One patient had developed progressive disease with wall thinning and cavity dimension increase. Two patients had developed echocardiographic LVH (in one, maximum left ventricular wall thickness became $>30 \mathrm{~mm}$; both also developed syncope). Two patients who remained LVH- developed clinical risk factors (one syncope and one abnormal exercise blood pressure response).

\section{LGE in LVH+ and LVH - patients}

Myocardial LGE was found in $12(80 \%)$ LVH+ patients, with variable extent (mean 15\%, range 3-48\%). LGE was also found in two (13\%) $\mathrm{LVH}-$ patients with limited extent $(3.6 \%)$. Both of these patients and one other LVH- patient were found to have regional hypertrophy by cine CMR despite normal echocardiography. These patients had abnormal Q waves and ST segment abnormalities. All the LVH+ patients also had abnormal ECGs. No other LVH- patient had an abnormal ECG.

LGE and clinical risk

The extent of LGE was associated with clinical risk factors for sudden death $(\geqslant 2 v \leqslant 1$ risk factors, $21 \% v 7 \%, \mathrm{p}=0.02)$ and total left ventricular mass $(r=0.56, \mathrm{p}<0.001)$ and was inversely associated with ejection fraction $(r=-0.58$, $\mathrm{p}<0.001)$.

\section{LGE within families}

Three families had more than four gene positive members, which illustrate the correlation of LGE, phenotype, and phenotype progression.

Family H805 (Arg 145Trp) (fig 1) has late onset, low risk disease. No patient has any risk factors for sudden death. No phenotype development was detected over three years of follow up. Individual 4, the proband, has the most LVH and it is associated with outflow tract obstruction and some $(6 \%)$ myocardial LGE. Individuals 2 and 3 fulfil proposed familial criteria for HCM with abnormal ECGs but normal echocardiograms. CMR detected LVH in both individual 2 and 3 and limited LGE in individual $3(3 \%)$.

In family H15 (Argl62Gln) (fig 2) the brother (proband) died suddenly aged 18 years and post mortem examination found extensive myocardial fibrosis and asymmetric hypertrophy. Over 12 years' follow up, individual 4, a male, developed LVH, which progressed to $>30 \mathrm{~mm}$ and an abnormal blood pressure response to exercise; 13\% LGE was found in the hypertrophied region of the anterior septum. His older sister had phenotype development with an abnormal ECG and an abnormal blood pressure response to exercise. CMR showed mild LVH and 3\% LGE.

Family H136 (Argl86Gln) (fig 3) has two patients who died in their 40s of heart failure (progressive disease). Individual 3 developed LVH and syncope over six years. CMR showed $11 \%$ LGE. Over 14 years' follow up, individual 4 developed LVH $>30 \mathrm{~mm}$, an abnormal exercise test, and subsequently wall thinning and left ventricular dilatation. CMR showed very extensive LGE of $48 \%$.

\section{Segmental LGE and regional hypertrophy}

As regional wall thickness increased, LGE was more prevalent $(\mathrm{p}<0.0001$ for trend $)$ and more extensive $(r=0.98$, $\mathrm{p}=0.001)($ fig 4$)$.

\section{DISCUSSION}

Familial genotype-phenotype studies indicate that disease causing sarcomeric gene mutations account for only some of 


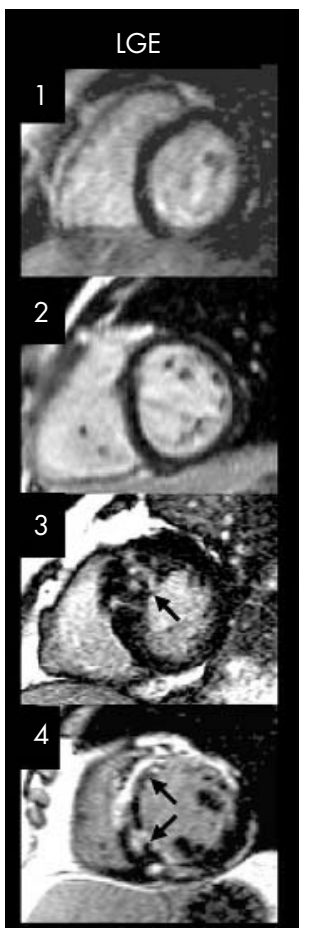

$\begin{array}{ll}11 \sigma^{\top} & \\ \text { EF } & 76 \% \\ \text { Mass } & 51 \\ \text { Cine/LGE } & n / 0 \% \\ \text { Echo } & n \\ \text { ECG } & n \\ 22 \text { + } & \\ \text { EF } & 69 \% \\ \text { Mass } & 51 \\ \text { Cine/LGE } & 0 \% \\ \text { Echo } & n \\ \text { ECG } & n \\ 24 \sigma^{\top} & \\ \text { EF } & 80 \% \\ \text { Mass } & 112 \\ \text { Cine/LGE } & a b n / 11 \% \\ \text { Echo } & a b n \\ \text { ECG } & a b n \\ 42 \sigma^{\top} & \\ \text { EF } & 41 \% \\ \text { Mass } & 129 \\ \text { Cine/LGE } & a b n / 48 \% \\ \text { Echo } & \text { abn } \\ \text { ECG } & a b n \\ & \end{array}$

Figure 3 Progression of LGE in a family with more than four gene positive members. H136 (Arg186Gln), a family with disease that progressed from normal through hypertrophy to heart failure. There is significant LGE in hypertrophied myocardium and massive LGE in the patient with previous left ventricular hypertrophy $>30 \mathrm{~mm}$ and subsequent wall thinning.
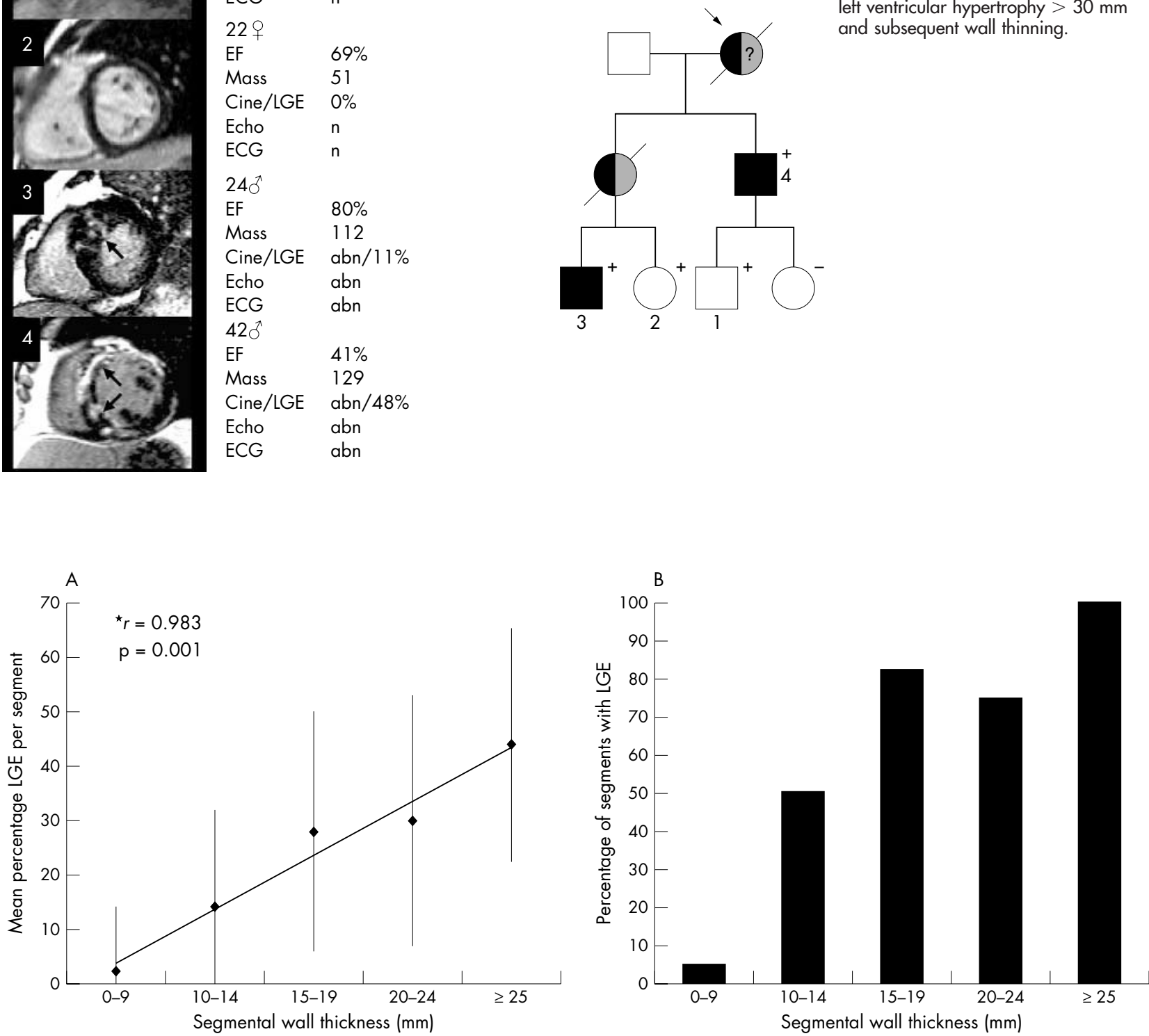

Figure 4 Combining the 480 myocardial segments from the 30 patients, the (A) prevalence and (B) extent of LGE increases with increasing segmental wall thickness.

the characteristic phenotypic variation in HCM. ${ }^{18}$ Studying a cohort with disease causing mutations of a single gene reduces the contribution of the sarcomeric mutation to phenotypic heterogeneity and allows investigation of patients before phenotypically declared disease.

Myocardial fibrosis plays an important part in end stage disease as shown by necropsy studies $^{819}$ but the role of fibrosis in developing disease is not understood because there has been no sensitive in vivo quantification technique. A greater understanding of phenotype development is a major aim in HCM because, although the genetic basis of HCM has been largely elucidated, the mediators between genetic defect and myocardial abnormality, and hence risk, are only now being investigated. ${ }^{\circ}$
LGE CMR provides a means of quantifying fibrosis in HCM. Gadolinium-DTPA is an extracellular tracer. After an intravenous bolus it is known that fibrosis from myocardial infarction appears enhanced by the highly sensitive inversion-recovery CMR technique late after injection. ${ }^{20}$ LGE is also found in $\mathrm{HCM}^{11}$ and has been found to represent regions of interstitial expansion and fibrosis. ${ }^{12}{ }^{21}$ It has been shown that the extent of LGE is associated with clinical risk factors for sudden death and the presence of progressive disease. ${ }^{10}$

The data presented in this paper support the clinical perspective that myocardial fibrosis, detected as LGE, may play an important part in HCM phenotype development. With the use of CMR to define the presence or absence of $\mathrm{LVH}$, regions of LGE were found in 14 of $18(78 \%)$ patients 
with LVH but in no patient without LVH, suggesting that regions of fibrosis occur only after the development of LVH. This is backed up by the strong but imperfect relation of segmental LGE presence and extent with segmental wall thickness (fig 4). In addition, the total extent of LGE was higher in patients with more risk factors for sudden death-a cohort in whom necropsy studies suggest that advanced myocardial abnormalities can be expected. The within-family data (for example, the family in fig 3), where clinical follow up had documented progressive myocardial hypertrophy and risk factor development, and in one patient subsequent thinning and left ventricular impairment show progressive LGE, even when the wall is thinning. These data suggest that the extent of LGE found in a patient, by being a marker of global myocardial abnormality, may be an indicator of a patient's risk at the time of measurement, reflecting the influence of all contributing factors, both genetic and environmental. Of course, the relation between extent of fibrosis and events may be complex and we hypothesise that the rate of LGE development may also be importantextensive LGE at a young age may carry more significance than a similar degree of LGE in an older patient.

A further implication of the study is that in patients with a TNNI3 mutation, the detection of LGE by CMR is not useful for screening for early disease before hypertrophy is present. Indeed, the study emphasises the known importance of ECG abnormalities in early disease ${ }^{22} 23$ because the three patients who were LVH - by echocardiography but LVH+ by CMR had abnormal ECGs. But the finding of a high incidence of LGE $(78 \%)$ in genotyped HCM is preliminary suggesting that LGE may be a highly specific marker of myopathy, although it will not be $100 \%$ sensitive, and may provide a useful technique for distinguishing HCM from other forms of non-myopathic LVH such as compensatory or physiological forms.

A limitation of this study is that we attempted to determine the genetic contribution to HCM but it is clear that there is considerable phenotype heterogeneity with different TNNI3 mutations, so some within-family analysis is presented. There may also be a genetic influence from linked and unlinked genetic modifiers. The number of patients is limited in this study and generalising the results to other gene mutations may need caution. This study brings together a novel technique for myocardial phenotyping, LGE CMR, with a population of patients with HCM who are well characterised both genetically and clinically. Further such studies are needed because LGE CMR has great potential to provide new insights into pathogenesis and disease expression in HCM.

\section{ACKNOWLEDGEMENTS}

Professor McKenna (chair holder), Dr James Moon (Junior Research Fellowship), and the Centre for Advanced Magnetic Resonance in Cardiology (CAMRIC) were supported by the British Heart Foundation. Research support was also received from CORDA and Siemens Medical Solutions.

\section{Authors' affiliations}

J C Moon, G C Smith, A G Elkington, S K Prasad, D J Pennell, Centre for Advanced Magnetic Resonance in Cardiology (CAMRIC), Royal Brompton Hospital, London, UK
J Mogensen, P M Elliott, W J McKenna, Cardiology in the Young, The Heart Hospital, University College London Hospitals Trust, London, UK

\section{REFERENCES}

1 Maron BJ, McKenna WJ, Danielson, et al. American College of Cardiology/European Society of Cardiology clinical expert consensus document on hypertrophic cardiomyopathy. J Am Coll Cardiol 2003;42:1687-713.

2 Richardson P, McKenna W, Bristow M, et al. Report of the 1995 World Health Organization/International Society and Federation of Cardiology task force on the definition and classification of cardiomyopathies. Circulation 1996;93:841-2.

3 Anderson KR, Sutton MG, Lie JT. Histopathological types of cardiac fibrosis in myocardial disease. J Pathol 1979;128:79-85.

4 St John Sutton MG, Lie JT, Anderson KR, et al. Histopathological specificity of hypertrophic obstructive cardiomyopathy: myocardial fibre disarray and myocardial fibrosis. Br Heart J 1980:44:433-43.

5 Marian AJ. Modifier genes for hypertrophic cardiomyopathy. Curr Opin Cardiol 2002; 17:242-52.

6 Tsybouleva N, Zhang L, Chen S, et al. Aldosterone, through novel signaling proteins, is a fundamental molecular bridge between the genetic defect and the cardiac phenotype of hypertrophic cardiomyopathy. Circulation 2004; 109:1284-91

7 Varnava AM, Elliott PM, Mahon N, et al. Relation between myocyte disarray and outcome in hypertrophic cardiomyopathy. Am J Cardiol 2001;88:275-9.

8 Varnava A, Elliott PM, Sharma S, et al. Hypertrophic cardiomyopathy: the interrelation of disarray, fibrosis, and small vessel disease. Heart 2000;84:476-82.

9 Becker AE. Cardiomyopathies with particular reference to the diagnostic relevance of endomyocardial biopsies. Wien Klin Wochenschr 1988; 100:787-91.

10 Moon JCC, McKenna W, McCrohon JA, et al. Towards clinical risk assessment in hypertrophic cardiomyopathy with gadolinium cardiovascular magnetic resonance. J Am Coll Cardiol 2003;41:1561-7.

11 Choudhury L, Mahrholdt H, Wagner A, et al. Myocardial scarring in asymptomatic or mildly symptomatic patients with hypertrophic cardiomyopathy. J Am Coll Cardiol 2002;40:2156-64

12 Moon JC, Reed E, Sheppard M, et al. The histological basis of myocardial hyperenhancement by gadolinium cardiovascular magnetic resonance in hypertrophic cardiomyopathy. J Am Coll Cardiol 2004;43:2260-4.

13 Mogensen J, Murphy RT, Kubo T, et al. Frequency and clinical expression of cardiac troponin I mutations in 748 families with hypertrophic cardiomyopathy. J Am Coll Cardiol 2004;44:2315-25.

14 Mogensen J, Kubo T, Duque M. Idiopathic restrictive cardiomyopathy is part of the clinical expression of cardiac troponin I mutations. J Clin Invest 2003;111:209-16.

15 Mogensen J, Bahl A, Kubo T, et al. Comparison of fluorescent SSCP and denaturing HPLC analysis with direct sequencing for mutation screening in hypertrophic cardiomyopathy. J Med Genet 2003;40:E59.

16 Elliott PM, Gimeno BJ, Mahon NG, et al. Relation between severity of leftventricular hypertrophy and prognosis in patients with hypertrophic cardiomyopathy. Lancet 2001;357:420-4.

17 Cerqueira MD, Weissman NJ, Dilsizian V, et al. Standardized myocardial segmentation and nomenclature for tomographic imaging of the heart: a statement for healthcare professionals from the cardiac imaging committee of the council on clinical cardiology of the American Heart Association. Circulation 2002;105:539-42.

18 Marian AJ, Roberts R. The molecular genetic basis for hypertrophic cardiomyopathy. J Mol Cell Cardiol 2001;33:655-70.

19 Davies MJ, McKenna WJ. Hypertrophic cardiomyopathy: pathology and pathogenesis. Histopathology 1995;26:493-500.

20 Wu E, Judd RM, Vargas JD, et al. Visualisation of presence, location, and transmural extent of healed $Q$-wave and non- $Q$-wave myocardial infarction. Lancet $2001 ; 357: 21-8$.

21 Kim RJ, Judd RM. Gadolinium-enhanced magnetic resonance imaging in hypertrophic cardiomyopathy: in vivo imaging of the pathologic substrate for premature cardiac death? J Am Coll Cardiol 2003;41:1568-72.

22 Al-Mahdawi S, Chamberlain S, Chojnowska L, et al. The electrocardiogram is a more sensitive indicator than echocardiography of hypertrophic cardiomyopathy in families with a mutation in the MYH7 gene. Br Heart J 1994;72:105-11.

23 McKenna WJ, Spirito $P$, Desnos $M$, et al. Experience from clinical genetics in hypertrophic cardiomyopathy: proposal for new diagnostic criteria in adult members of affected families. Heart 1997;77:130-2. 